\title{
MIRAS AS PROBES OF THE GALACTIC BULGE
}

\author{
S.M.G. HUGHES, R.M. CATCHPOLE \\ Royal Greenwich Observatory, Cambridge, United Kingdom \\ P.A. WHITELOCK \\ South African Astronomical Observatory, South Africa \\ AND \\ M.W. FEAST \\ Astronomy Dep., University of Cape Town, South Africa
}

\section{Introduction}

Mira variables span a wide range of stellar populations and have a welldefined $K, \log P$ relation (e.g. Feast et al 1989; Hughes \& Wood 1990), so can be used to map out the 3-D distribution of the bulge. Using IRASselected Miras, Whitelock \& Catchpole (1992) showed for the first time from individual stellar distances that the bulge had a bar-like structure, but there is some uncertainty as to whether they obey the same $K, \log P$ relation as optically selected Miras. Hence the need for confirmation from an optical Mira survey.

\section{Observations}

These optical bulge Miras were detected from APM scans of IVN (I-band) plates taken by the AAO UK Schmidt telescope, over the period of June 1990 to August 1996, covering four bulge fields, spanning $l=-9$ to +9 , and $b=-4$ to -15 . A total of 833 Mira candidates were detected, 291 of which were observed in the infrared $(J, H, K, L)$, using the SAAO $1.9 \mathrm{~m}$.

\section{Bulge LPVs: 3D distributions}

We first measure the amount of extinction to each Mira by assuming that they are all oxygen-rich stars (of 45 spectra obtained, only one is carbonrich), and that they obey the same $(J-K)_{P}, \log P$ relation as their cousins 
in the LMC (Hughes 1992), so the colour excess will simply be $E(J-K)=$ $(J-K)_{\text {observed }}-(J-K)_{P}$. We then obtain distances to the most likely Mira candidates via the LMC $K, \log P$ relation (Feast et al. 1989), where we assume an LMC distance modulus of 18.55 magnitudes (e.g. van Leeuwen et al. 1997).

A least squares fit to the bulge Mira distribution (those with distances between 5.4 and $13 \mathrm{kpc}$, to avoid confusion from the foreground and background Miras) yields an inclination of $\sim 35^{\circ}$ and a distance to the centre of the Mira bulge population of $\sim 8.2 \mathrm{kpc}$. Due to systematic errors arising from the assumptions about bulge membership and reddening corrections, both of these are lower limits. The effect of defining the bulge distribution by distance will artificially skew the least squares solution toward a zero slope solution. Nevertheless, a positive slope confirms the trend of decreasing mean distance with increasing longitude, as was found for the IRASselected Miras (Whitelock and Catchpole 1992). For the distances, applying the LMC colour-period relation to derive reddenings will overestimate these (and thus underestimate the distances), as it is known that galactic Miras have redder $(J-K)_{0}$ colours than those in the LMC (Whitelock et al. 1994 ) and that this is almost certainly a metallicity effect (Feast 1996). No evidence has so far been found for a dependence of the distribution of the bulge Miras on period.

\section{Future Work}

As we have measured only lower limits, this report merely shows the potential usefulness of these data. Our final analysis will measure the true tilt via sophisticated fits which model the distribution of the bar, and use a better method to estimate the interstellar extinctions via a new $K-L, \log P$ relation for the LMC Miras, as this will be less affected by metallicity. We are also identifying more Miras in an additional bulge field, and continue to obtain IR photometry and FLAIR spectroscopy of a larger sample, the latter to search for any kinematic effects as a function of period (age).

\section{References}

Feast, M.W., 1996, MNRAS, 278, 11

Feast, M.W, Glass, I.S., Whitelock, P.A., Catchpole, R. 1989 MNRAS, 241, 375

Hughes, S.M.G. 1992, IAU Coll. 139, eds. J.M.Nemec and J.M.Matthews (CUP), p192. Hughes, S.M.G. \& Wood, P.R. 1990, AJ, 99, 784

van Leeuwen, F., Feast, M. W., Whitelock, P. A., Yudin, B. 1997 MNRAS 287, 955

Whitelock, P.A. \& Catchpole, R.M., 1992 in The Center, Bulge and Disk of the Milky Way, ed. Blitz, L. (Kluwer: Dordrecht), p.103

Whitelock, P.A., et al., 1994, MNRAS, 267, 711 